\title{
Past, present and future perspectives of genetic therapy in gliomas
}

\author{
Tobias Alécio Mattei, Ricardo Ramina, Marcos Tatagiba, Paulo Henrique Aguiar
}

Department of Neurosurgery, Sao Paulo Medical School, Brazil.

High-grade gliomas are relatively frequent in adults and consist in the most malignant form of primary brain tumor. They are resistant to standard treatment modalities such as surgery, radiation, and chemotherapy, being fatal within 1 to 2 years of onset of symptoms. Owing to the promising practical clinical benefits that can be expected for the near future, an exposition of the basic issues in genetic therapy of gliomas seems timely. In this article we intend to provide a general review that covers the most important genes already studied as possible agents for genetic therapy in gliomas. In a critical analysis we intend to expose and discuss anti-tumoral mechanisms and therapeutical results of studies with the following class of genes: prodrug activation systems, apoptosis-related genes, anti-angiogenic factors genes, radiosensitization genes, chemosensitization genes, apoptosis-related genes and immunogenes. Finally we discuss the historical importance, actual role and further developments that can be expected from each of these class of agents for the future of genetic therapy of gliomas.

Key Words: Brain tumors, genetic therapy, gene transfer, high-grade gliomas

\section{Introduction}

Gliomas in adults are devastating diseases, with very poor survival despite their lack of distant metastases. The best avaiable treatments, such as surgical resection and radiotherapy have been only temporarly successful, once recidives are almost always present, being fatal within 1 to 2 years of onset of first symptoms. Two factors promoting the use of gene therapy for gliomas are the failure and toxicity of conventional thera- pies and the identification of the genetic abnormalities that contribute to the malignancy of gliomas.

Uncontrolled cellular proliferation, lack of apoptosis, invasion, and angiogenesis are among the biologic processes that render these tumors both aggressive and difficult to treat. Until recently, our knowledge of this type of tumor has mainly been based on chromosomal and molecular studies performed in the last two decades. This has increased tremendously with the advent of new technologies, in particular expression arrays for simultaneous analysis of thousands of genes.

During the malignant progression of gliomas several tumor suppressor genes are inactivated, and numerous growth factors and oncogenes are overexpressed progressively. Consequently, gene therapy of gliomas may aim at molecular interference with 'gain of function' genes (oncogenes) or replacement of 'loss of function' genes (tumor suppressor genes). Such approaches require transgene expression in whole tumor cell populations (if not other mechanisms come into play) which cannot be achieved with current vector systems. Hence other strategies have been pursued which may be independent of genes actually involved in tumorigenesis. Microbial genes (e.g. herpes simplex virus thymidine kinase) may be transferred into the tumors allowing for prodrug activation (e.g. ganciclovir). Furthermore, that frequently involves ex vivo transfection of autologous tumor cells with such genes. ${ }^{[1]}$

Malignant gliomas have been chosen for the first clini- 
cal studies on novel gene therapy approaches because these tumors are non-metastatic and develop on the largely postmitotic background of normal glial and neuronal tissue. Several molecular strategies have been tested, either in animal models or clinical trials: prodrug activating systems, introduction of tumor suppressor or cell-cycle-related genes, inhibition of growth factors and/ or their receptors, inhibition of neovascularization, immunomodulatory maneuvers, oncolytic viruses, inhibition of matrix metalloproteinases, induction of local expression of toxic agents and sensitization of tumors to chemotherapeutic agents and radiotheraphy. ${ }^{[2,3]}$

Critical evaluation of gene transfer and therapy studies has led to the conclusion that, even using identical vectors, the anatomical route of vector can dramatically affect both the efficiency of tumor transduction and its spatial distribution, as well as the extent of intratumoral and intracerebral transgene expression. The safety and efficiency of these therapeutic systems in humans have been availed by several controlled preclinical and clinical therapeutic trials. ${ }^{[4-6]}$

\section{Genes}

\section{Prodrug Activation Systems HSV-TK/Ganciclovir}

Tumor cell transduction with herpes simplex virus thymidine kinase (HSV-TK) gene and treatment with ganciclovir (GCV) is the more widely studied cancer gene therapy. ${ }^{[7-11]}$ HSV-TK converts the pro-drug ganciclovir (GCV) into a toxic nucleotide analogue, the incorporation of which into cellular DNA blocks cell proliferation. Following repetitive ganciclovir (GCV) intraperitoneal or intravenous injection, effective killing of glioma cells in mouse brain is observed [Figure 1]. In vivo studies relate a significant regression of the tumors in $50-80 \%$ of the rats treated with this therapy modality as compared with control animals. In some animals tumors completely disappeared after this treatment protocol and mice were considerated are completely cured. ${ }^{[12]}$ [Figure 2]

Posterior analysis of the inoculated tissue showed that transduced glioma cells are found far from the implantation site, potentiating the induction of the therapeutic effect, what was explained as result of what was called "bystander effect". The "bystander effect" is a paradigm

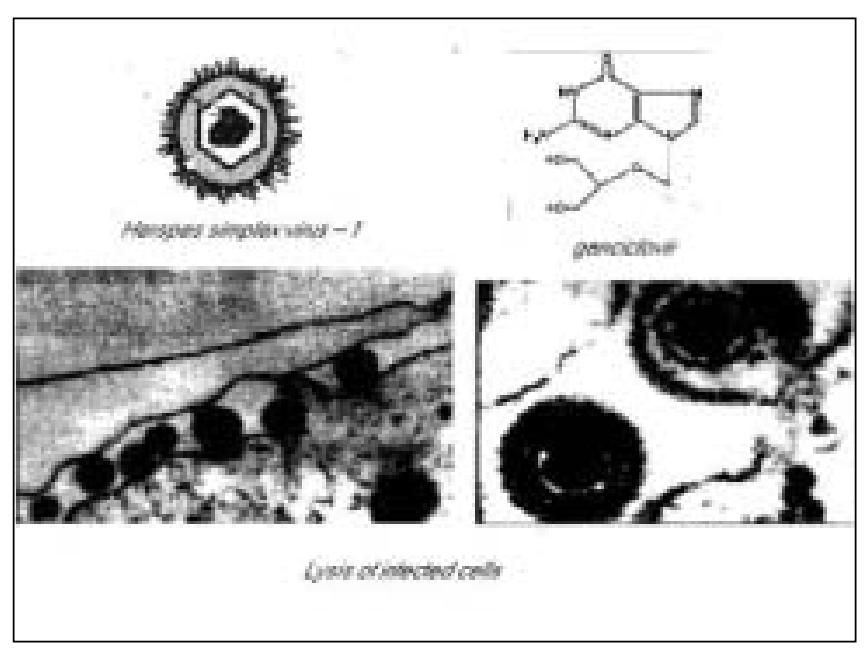

Figure 1: Therapy with HSV-tk oncolytic virus and further ganciclovir injection

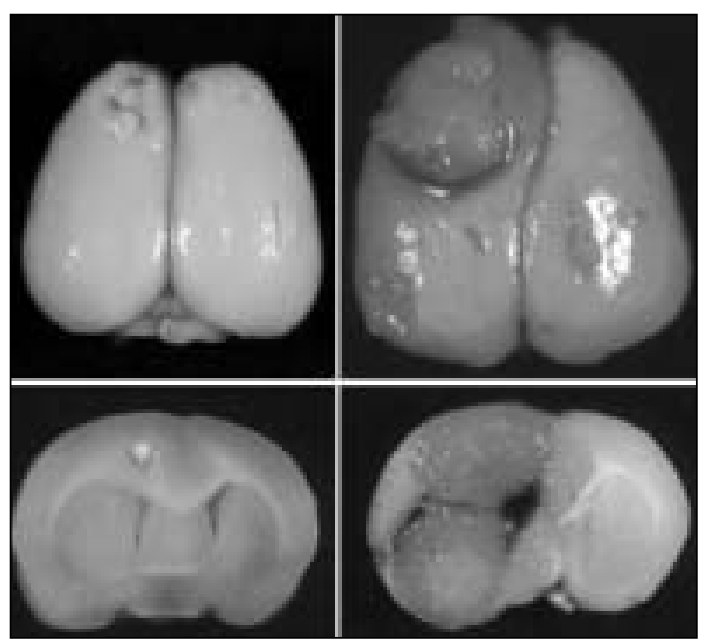

Figure 2: Brains of rats injected in with $9 \mathrm{~L}$ cells of gliosarcoma. Left - "HSV/tk ganciclovir Group" -Only an empty cavity is observed at the site where before there was the tumour. Right - "Control Group" - The growing tumour occupies almost all the left frontal lobe of the rat

of the suicide gene therapy and consists in a phenomenon of bystander cell killing, that is to say, the death of adjacent tumor cells not transduced with the HSV-TK gene after treatment with the antiviral drug, ganciclovir (GCV). Evidence from quantitative in vitro assays of glioma cell lines suggest that both murine and human gliomas are similar in expressing high sensitivity to the bystander effect. In vitro studies revealed that the presence of only $5 \%$ of HSV-TK-expressing transduced cells in the culture results in more than $90 \%$ tumor cell death/ stasis after addition of GCV. ${ }^{[13,14]}$

Several lines of evidence support gap junction intercellular communication (GJIC) as important in the bystander effect. In vitro metabolic assays, performed with GCV in the medium, indicate that tumor are more re- 
duced when culture conditions support cell-cell contact of parental and HSV-TK-transduced cells. Additionally, a double dye transfer assay showed that cell communication through the gap junction is greatest for glioma, less for melanoma, and much less for colorectal carcinoma cell lines. In vitro metabolic assays with mixtures of $\mathrm{TK}+/ \mathrm{TK}$ - homologous tumor cells confirmed that glioma cell lines are more susceptible to bystander killing than melanomas.

Finally, the importance of a gap junction protein, such as connexin-43, in facilitating the bystander effect was demonstrated in a HT29 low-GJIC cell line study. When the TK-nontransduced cell population expressed connexin-43, a better bystander kill was achieved compared to the parental counterpart. ${ }^{[15]}$

\section{Thymidine kinase gene therapy modifications Connexin}

As previously exposed, Connexin (Cx)-dependent gap junctions between cells facilitate the intercellular spread of TK-activated GCV, thereby creating a bystander effect that improves tumor cell killing. However, tumor cells often have reduced connexin expression, thus thwarting bystander killing and the effectiveness of TK/GCV gene therapy. To improve the effectiveness of this therapy, some authors studied an HSV vector (TOCX) expressing $\mathrm{Cx} 43$ in addition to TK in U-87 MG human glioblastoma cells in vitro and in vivo. ${ }^{[8,13]}$ In vivo experiments in which U-87 MG tumoral cells were preinfected with HSV vector (TOCX) expressing $\mathrm{Cx} 43$ in addition to TK injected into the flanks of nude mice showed complete cures of all animals following GCV treatment, whereas animals with non-infected cells (control group) uniformly died from the tumor. The TOCX injection into U-87 MG intracranial tumors resulted in prolonged survival of the host animals when compared with the animals treated only with HSV-TK/GCV therapy. Together, these results suggest that the combination of TK and Cx may be beneficial for the treatment of high-grade gliomas.

\section{Sustained release silicone formulation}

In order to enhance the cytotoxic effects of herpes simplex virus thymidine kinase suicide gene therapy, some researchers developed a silicone formulation of ganciclovir (GCV-pellet) of $1 \mathrm{~mm}$ in length and in diam- eter containing a total amount of $0.15 \mathrm{mg}$ of GCV. In vitro experiments demonstrated that GCV was gradually released over a period of 7 days. The results confirmed that sustained release of a low and effective GCV dose with the silicone formulation significantly prolonged survival in combinations with HSV-TK expression if compared to intraperitoneal administration of GCV. Histological examination suggested that the treatment appears to be safe [Figure 3]. ${ }^{[16]}$

\section{P450/Cyclophosphamide}

Cyclophosphamide is an inactive prodrug which is converted by hepatic cytochrome P450 2B1 to cytotoxic metabolites which produce interstrand DNA cross-linking in a cell cycle-independent fashion. The limited ability of these metabolites to cross the blood-brain barrier contributes to the poor activity of cyclophosphamide against brain tumors.

It had been suggested that in situ transduction of tumor cells with the P450 2B1 gene -responsible for bioconverting the chemotherapic cyclophosphamide (CPA) into its active metabolites 4-hydroxyCPA/ aldophosphamide (AP) - using retroviral and adenoviral vectors could increase the sensitivity of $9 \mathrm{~L}$ gliomas cells

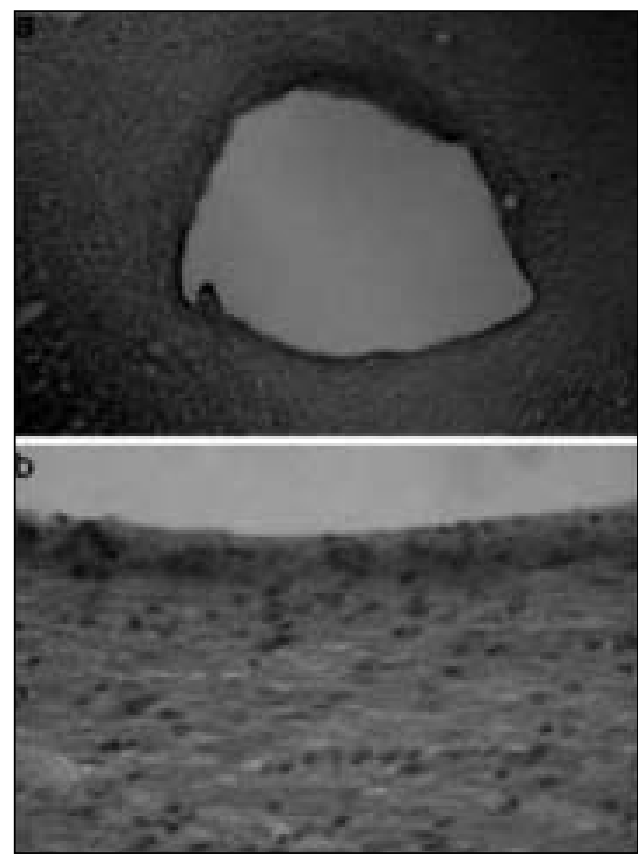

Figure 3: Histopathological sections of area adjacent to tumor that was injected with HSV/tk viral therapy with sustained release silicone formulation. (a) $H / E, x 10$, (b)

$H / E, x 40)$. No immunological or toxic reaction is observed in the normal areas adjacent to the cavity left by the tumour 
to cyclophosphamide and have a potential role in the therapy of malignant gliomas. In vivo studies showed that P450 2B1 gene transduction prolongs the survival of animals bearing intracerebral $9 \mathrm{~L}$ tumors treated with cyclophosphamide. ${ }^{[17]}$ More prolonged and higher concentrations of activated metabolites are generated by intraneoplastic injection compared with systemic administration of prodrug. ${ }^{[18]}$

\section{$\mathrm{Na}+/ \mathrm{l}-\mathrm{symporter}$}

Radioiodide concentrating activity in the thyroid, mediated by human $\mathrm{Na}+/ \mathrm{l}-$ symporter ( $\mathrm{hNIS}$ ), provides a mechanism for effective radioiodide treatment for patients who have invasive, recurrent, and metastatic thyroid cancers after total thyroidectomy.

Some attempts to develop hNIS gene transfer for radioiodide therapy in patients with malignant glioma have been tried. Altough more in vivo research is necessary, tumoral hNIS transduction through adenovirus vector followed by radioiodide therapy is a modality of genetic therapy that promisses to have great progress in the next years. ${ }^{[19]}$

\section{Folylpolyglutamyl synthetase}

Although antifolates are popular agents for use in chemotherapy, they display minimal toxicity against slow-growing tumors and are toxic to actively replicating cells in normal tissues. These drugs are converted intracellularly into polyglutamate derivatives by the enzyme folylpolyglutamyl synthetase (FPGS). Because tumors with high expression of FPGS often respond to nontoxic antifolate doses, it has been investigated whether augmenting tumoral FPGS activity by gene delivery would enhance tumoral antifolate sensitivity. The results suggest that FPGS gene delivery enhances the antifolate sensitivity of some glioma cell lines. However this approach still need further evaluation as a therapeutic strategy. ${ }^{[20]}$

\section{Anti-angiogenic factors}

Inhibition of angiogenesis has been considered among the most promising approaches to treat highly vascularized solid tumors such as high-grade gliomas. However chronic systemic delivery of therapeutic pro- teins, such as inhibitors of angiogenesis, present a number of difficult pharmacological challenges. The concept that targeted antiangiogenesis, using virally mediated gene transfer, represents a promising strategy for delivering this antiangiogenic factors. ${ }^{[21]}$

\section{Angiostatin, Endostatin, and IFN-alpha(1)}

Some researchers evaluated the effects of local production of three endogenous inhibitors of angiogenesis: angiostatin, endostatin, and interferon (IFN)-alpha(1), using stably transfected rat (9L) and human (GL15) glioblastoma cells on tumor vascularization and growth in an in vitro assay system based on the implantation of tumor cells into organotypic brain slice cultures.

Although all the three genes showed angiogenesis inhibitory effect, IFN-alpha demonstrated the most potent antiangiogenic effect in organotypic brain slice cultures. ${ }^{[20]}$ In vivo, after intracerebral implantation of such genetically modified high-grade glioma cells, IFN-alpha(1) caused a dramatic decrease in tumor volume revealed by magnetic resonance imaging and by postmortem histology. Mechanisms of this antitumor effect were most likely caused by the major antiangiogenic action of the cytokine, once IFN-alpha 1) expression provoked a pronounced decrease in blood vessel density, which was accompanied by extensive necrosis in the body mass of the tumors. ${ }^{[22]}$

\section{Platelet factor 4 (sPF4)}

Retroviral and adenoviral vectors that express a novel, secretable form of the antiangiogenic protein, platelet factor 4 (sPF4), have been constructed. It was showed that vector-mediated SPF4 transduction selectively inhibits endothelial cell proliferation in vitro, and results in hypovascular tumors that grow slowly in vivo. Additionally, tumor-associated angiogenesis is inhibited and animal survival is prolonged, following transduction of established intracerebral gliomas by an SPF4-expressing adenoviral vector. ${ }^{[21]}$

\section{p16 gene}

Recent studies have indicated that the loss of p16 is a frequent event in the progression of malignant gliomas. The loss of 16 promotes the acquisition of malig nant characteristics in gliomas, which are among the 
most angiogenic of all human tumors. High-grade gliomas can be distinguished from low-grade gliomas by the intense angiogenesis in addition to their frequent loss of $\mathrm{p} 16$.

Infection with a recombinant replication-defective adenovirus vector containing cDNA of wild-type p16 significantly reduced the expression of vascular endothelial growth factor, which is thought to be a pivotal mediator of tumor angiogenesis in p16-deleted glioma cells. Restoring wild-type p16 expression into p16-deleted glioma cells markedly inhibited angiogenesis induced by tumor cells in vivo. Furthermore, wild-type p16 inhibited neovascularization more potently than did wild-type p53 transfer. ${ }^{[23]}$

\section{Apoptosis-related genes}

\section{p-53 gene}

P-53 gene therapy is in clinical development for the treatment of various cancers. P-53 gene is thought to function abnormally in the majority of malignant gliomas, although it has been demonstrated to be mutated in only approximately $30 \% .10$ This fact has encouraged studies in which adenoviral transduction with wildtype human p53 has been investigated in an attempt to slow tumor cell growth. Some authors showed that multiple gene replacements with the simultaneous exposure to adenovirus containing p53 gene can produce additive effects in the treatment of glioma cell lines. ${ }^{[2]}$

\section{Rb gene}

The development of high-grade gliomas involves progressive inactivation of several tumor suppressor genes. Abnormalities of the retinoblastoma tumor suppressor gene are found in the majority of cancers, including at least $30 \%$ of malignant gliomas. In face of the evidence that restoration of the retinoblastoma protein function may have therapeutic application for gliomas it was evaluated the effect of the adenovirus transfer of the 3.2-kb cDNA of the Rb gene to human glioma xenografts implanted subcutaneously in nude mice. The findings provide direct evidence that inactivation of the retinoblastoma protein is a critical event in gliomas, and suggest that the restoration of wild-type retinoblastoma activityin these tumors through vector delivery gene theraphy may have great therapeutic utility. ${ }^{[25]}$

\section{TRAIL}

Tumor necrosis factor-related apoptosis-inducing ligand (TRAIL) induces apoptosis in a variety of transformed cell lines, but generally spares most normal cells. Transduction with an adenoviral vector expressing human TRAIL cDNA resulted in both direct glioma cell killing as well as a potent bystander effect through presentation of TRAIL by transduced normal cells. Administration of adenovirus containing TRAIL gene significantly prolonged survival of mice harboring intracerebral glioblastomas. Taken together, these data suggest that vector-mediated transduction of TRAIL may represent an effective strategy for high-grade glioma gene therapy. ${ }^{[26]}$

\section{FADD gene}

Fas/APO-1 (CD95), a cell surface cytokine receptor, triggers apoptotic cell death by specific agonist antibody, suggesting that Fas/APO-1 may be a promising target for treatment of tumors. It has been showed that treatment with anti-Fas antibody (FADD) effectively induced apoptosis in malignant glioma cell lines with high expression of Fas/APO-1 The retroviral transfer of FADD gene significantly enhanced the transduction efficiency and effectively inhibited both in vitro and in vivo survival of malignant glioma cells through induction of apoptosis. These findings suggest that the FADD gene is a novel and useful tool for the treatment of malignant gliomas. ${ }^{[27]}$

\section{Other apoptosis-related genes}

Some studies suggest that adenoviral vector-mediated delivery of other apoptosis-related genes may be also potentially useful in the gene therapy approach toward the treatment of human brain gliomas. Apoptosisrelated genes already studied are caspase-8, p33ING1, p73alpha, Bax, Apaf-1, caspase-9, IkappaBdN, NFkappaB, caspase-3, gas-1, Bcl-2, and $\mathrm{Bcl}-\mathrm{X}(\mathrm{L}){ }^{\left[{ }^{[28,29]}\right.}$

\section{Immunogenes}

Cancer immunogene therapy is based on vaccination with radiated, autologous tumor cells transduced with immunostimulatory genes. Several cytokines have been studied wheter as vaccine ex vivo transfection 
models or the classic in situ inoculation of the vector modified to carry the cytokine gene. ${ }^{[30-32]}$

The vaccination treatment using tumor cells genetically modified to express certain cytokines consist in the following steps. First glioma cells are cultured primarily from surgically resected tumor tissues of patients. Posteriorly, $\mathrm{n}$ vitro infection with a recombinant virus vector containing the gene of the cytokine is proceded, and lately, transduced cells are re-injected in the patient. Vaccination therapy induces specific activation of cytotoxic $T$ lymphocytes measured by cell-mediated cytotoxicity assay, suggesting the generation of a specific anti-tumor response and the potential for systemic immunity. This immunization results in regression of implanted cells as well as the original brain tumour. Mice cured of their intracerebral tumors by these vacciantion therapy also demonstrated protective immunity upon rechallenge, showing that these modality of therapy may have promising results in preventing the important problem of tumour recidives, almost always present in high grade gliomas. ${ }^{[33]}$

Several cytokines have been studied: IL-12, IL-6, IL4, IL-18, IFN-gamma, TGF-beta, TNF-alpha, GM-CSF, B7-2, TNF-alpha, LIF and LT. ${ }^{[34-37]}$ In conclusion, genetic immunothearpy has shown to be a promising potential both for treatment of established intracranial glioma through potentiatinon of antitumor immunity of the patient and for vaccination against subsequent intracranial glioma cell implantation.

\section{Chemosensitization genes}

\section{Cell cycle regulator WAF1/Cip1}

Studies have shown that the negative cell cycle regulator WAF1/Cip1 is often overexpressed in human gliomas and that WAF1/Cip1 overexpression renders glioma cells resistant to chemotherapy agents. It has been investigated whether down-regulation of WAF1/Cip1 could sensitize gliomas to chemotherapy. The results showed that the attenuation of WAF1/Cip1 expression initiated glioma cell death and sensitized glioma cells to apoptosis induced by 1,3-bis(2-chloroethyl)-1-nitrosourea and cisplatin. Thus, blocking WAF1/Cip1 production may serve as a useful chemosensitization regimen for treating glioma. ${ }^{[38]}$

\section{Cytosine deaminase/5-fluorocytosine}

5-Fluorouracil (5-FU) is a potent antimetabolite used for chemotherapy of gastrointestinal (GI), breast, and head and neck malignancies. Despite initial excitment, therapeutic index of 5-FU has showed to be very poor. It is unclear whether this lack of utility is due to problems with drug delivery or inherent insensitivity. Adenovirus (Ad) vector-mediated cytosine deaminase (CD)/ 5-fluorocytosine (5-FC) gene therapy has been proposed as a potential technique to overcome pharmacokinetic issues associated with systemic $5-\mathrm{FU}$ and is particularly well suited to use with tumors in which local control is paramount, such as malignant gliomas. Results of clinical studies in human patients with high-grade gliomas confirm the previous findings in rat models, demonstrating the potential clinical utility of adenovirus 5FC gene therapy for gliomas. ${ }^{[39]}$

\section{Radiosensitization genes}

\section{Bax gene}

It has been proposed that adenovirus-mediated transfection of proapoptotic Bax gene could enhance the cytotoxicity of radiotherapy (RT) in RT-refractory glioma cells. Results of in vivo experiments showed that apoptotic death may be enhanced by the combination of the treatment with adenovirus containing Bax gene (Ad-Bax) and RT. Remarkably, combined treatment induce regression of tumors in mice. In animals injected with subcutaneous tumors (D54 MG line), levels of apoptosis after RT alone, Ad/Bax alone, or the combination were, respectively, $12.3 \%, 32.1 \%$, and $78.5 \%$. These results confirms that $\mathrm{Ad} / \mathrm{Bax}$ synergistically radiosensitizes glioma, with a seemingly favorable therapeutic index. ${ }^{[40]}$

\section{p53 gene}

As exposed before there are studies, even in advanced clinical trial stage, in which adenoviral transduction with wild-type human p53 demonstrated to slow tumor cell growth. Investigations with p53 gene have also suggested that adenoviral vector-mediated expression of human wildtype p53 could not only slow tumor cell growth but also enhance the radiosensitivity of malignant glioma cells that express native wild-type p53. 
It was demonstrated that RT2 tumour cells express native rat wild-type p53 before the transduction and markedly overexpressed human p53 following adenoviral p53 transduction. P53 transduction followed by radiation resulted in marked decreases in RT2 cell survival and a increase in apoptosis at radiation doses from 2 to 6 Gy. Animals receiving cranial radiation after intracerebral implantation with RT2 cells previously transduced with p53 survived significantly longer than control animals. ${ }^{[41]}$ These results support a new perspective in the p53 genetic therapy, showing the ability to enhance the radiosensitivity of malignant glioma cells that express wild-type p53 by using adenoviral transduction to induce overexpression of $p 53$. This offers new hope for the p53 viral mediated genetic therapy as a sucessful therapeutic strategy, not only in human gliomas that express mutant p53, but also in those that express wild-type p53.

\section{Cytokines}

Some cytokine vaccination (GM-CSF, IL-4, IL-12) therapies have shown to have not only primary immunity generation against the tumour, but also an important radiosensitization effect. In some studies with tumors treated with vaccination therapy and posteriorly irradiation, about $80-100 \%$ of the glioma-bearing mice was cured. ${ }^{[42-44]}$

\section{Other genes}

\section{Carcinoembryonic antigen (CEA)}

CEA has potent antitumor activity against gliomas in vitro, as well as in orthotopic U87 animal models. Retrovirus mediated infection of a variety of glioblastoma cell lines including U87, U118, and U251 with CEA gene resulted in significant cytopathic effect consisting of excessive syncycial formation and massive cell death at 72-96 $\mathrm{h}$ from infection.

Mice with established xenografts tumors treated with a total dose of $8 \times 10$ (7) plaque forming units of Measles Virus-CEA (MV-CEA) had statistically significant regression of tumors and prolongation of survival in MV-CEA treated animals compared with the control groups. Histological examination of brains of MV-CEA-treated animals revealed complete regression of the tumor with the presence of a residual glial scar and reactive changes, mainly presence of hemosiderin-laden macrophages. ${ }^{[45]}$

\section{Urokinase-type plasminogen activator}

Urokinase-type plasminogen activator (UPA) and its receptor (UPAR) play an important role in the invasiveness of gliomas and other infiltrative tumors. In glioma cell lines and tumors, high grade correlates with increased expression of UPAR and UPA. Downregulation of UPAR and UPA by delivery of antisense sequences of UPAR and UPA in a single adenoviral vector (Ad), Ad-uPAR-uPA have been proposed as an method for genetic therapy in gliomas. Subcutaneous injections of bicistronic antisense constructs into established tumors (U87 MG) caused marked regression of these tumors. ${ }^{[46]}$ These results support the therapeutic potential of targeting the individual components of the UPAR-uPA system by using a single adenovirus construct for the treatment of malignant gliomas.

\section{Linamarase}

A new strategy for cancer gene therapy has been developed using a plant gene that encodes the enzyme, linamarase. This enzyme hydrolyzes cyanogenic glucoside substrate, linamarin, into glucose, acetone and cyanide. Retroviral vectors that carry linamarase as a potential killer-suicide gene caused a marked sensitization to the innocuous substrate, linamarin, followed by cell death. A study has demonstrated that this system can eradicate very large intracerebral gliomas in vivo helped by a cyanide bystander effect. Animals showed total regression of the glioma by magnetic resonance imaging (MRI) and do not showed other appreciable toxic effects. ${ }^{[47]}$

\section{Clinical Trials}

The most studied candidates for gene therapy, which are in advanced stages of clinical trials are: the prodrug activating system herpes simplex thymidine kinase (HSVtk)/ganciclovir (GCV), utilizing either retrovirus vector producer cells or adenovirus vectors, adenovirus mediated p53 gene transfer, adenovirus mediated IFN-beta gene transfer and 
studies with oncolytic therapy with herpes virus or adenovirus vectors. Other vectors and genes previously discussed are still in cell or animal protocols investigation stage.

The therapeutic potential of a designed bicistronic Moloney vector (pLIL-2-TK), combining the expression of a suicide gene (thymidine kinase, tk) with an immunomodulatory gene (human interleukin 2, IL-2) has also been tested in a small pilot sutdy in humans. Results showed evidence of transgene activity in the treated tumours and good results in regression of the tumours is presented. ${ }^{[48]}$

The most advanced study with p53 gene is a phase I clinical trial of p53 gene therapy using an adenovirus vector (Ad-p53) has been undertaken. Fifty-eight patients underwent a two-stage approach. In stage 1, Ad-p53 was stereotactically injected intratumorally via an implanted catheter. In stage 2 , the tumor-catheter was resected en bloc, and the postresection cavity was treated with Ad-p53. In all 15 patients, exogenous p53 protein was detected within the nuclei of astrocytic tumor cells. Exogenous p53 transactivated p21CIP/WAF and induced apoptosis. However, transfected cells resided on average within $5 \mathrm{~mm}$ of the injection site. The clinical toxicity was minimal and a maximum-tolerated dose was not reached. Although anti-adenovirus type 5 (Ad5) titers increased in most patients, there was no evidence of systemic viral dissemination. ${ }^{[49]}$ The results of this clinical trial showed that although p53 adenoviral transfection is a promisse therapy, at the dose and schedule evaluated, transduced cells were only found within a short distance of the injection site, revealing that widespread distribution of this agent remains a significant goal.

There is a ongoing phase $\mathrm{I} / \mathrm{I}$ clinical study in adult patients with recurrent high-grade glioma which aimed to evaluate biological safety, maximum tolerated dose and antitumor efficacy of a cytokine vacination model, using a genetically modified replication-disabled Semliki forest virus vector (SFV) that carries the human interleukin 12 (IL-12) gene. ${ }^{[50]}$

Several other Phase I and II clinical studies in patients with recurrent malignant glioma have shown a favorable safety profile and some efficacy of retroviruses
(RV) mediated gene therapy. ${ }^{[51]}$

More than 300 patients with glioma have been already treated in clinical trials with oncolytic viruses, and in most cases virus was administered directly into the tumor. Total intratumoral doses of up to $2 \times 10$ (12) virus particles were well tolerated, and Phase I clinical studies have demonstrated low to moderate toxicity grom the clinical use of oncolytic adenovirus or HSV-1 virus, either in the brain or in the rest of the body. Variable levels of gene transfer and in some cases anti-tumour effect have been observed. ${ }^{[52]}$

\section{Conclusions}

From the results until the present it is clear that gene therapy strategies for gliomas are quite promising but more critical research is required, mainly in the vector field. Although much effort has been put into this area of research, neuro-oncologists are still awaiting a vector system that allows selective and efficient tumor cell transduction. Further major advancements in virus designs, application modalities and understanding of the interactions of the host's immune system with the virus are clearly needed before oncolytic virus therapy of malignant brain tumours can be introduced to clinical practice.

Major steps to improve gene transfer into central nervous system and efficacy of gene therapy for malignant brain tumors include: 1) design of more effective vector systems; 2) development of new or improved prodrug/suicide systems, gene replacement approaches, or strategies targeting the immune response or tumor angiogenesis; 3) study of new techniques to enhance delivery of genetic vectors into brain tumors and for monitoring gene delivery into tumors.

Furthermore, clinical studies did not address main limiting factors for in vivo gene therapy, such as insufficient gene transfer rates to the tumor with used local delivery modalities, and resulting inability of a particular transgene-prodrug system to confer permanently eradicating cytotoxicity to the whole neoplasm. The ultimate molecular therapy will probably involve the application of multiple simultaneous (combinatorial) therapeutic modalities. 


\section{References}

1. Hamel W, Westphal M. Gene therapy of gliomas. Acta Neurochir Suppl 2003;88:125-35.

2. Eck SL, Alavi JB, Alavi A, Davis A, et al. Treatment of advanced CNS malignancies with the recombinant adenovirus H5.010RSVTK: A phase I trial. Hum Gene Ther 1996;7:1465-82.

3. Germano IM, Fable J, Gultekin SH, Silvers A. Adenovirus/herpes simplex-thymidine kinase/ganciclovir complex: preliminary results of a phase I trial in patients with recurrent malignant gliomas. J Neurooncol 2003;65:279-89.

4. Prados MD, McDermott M, Chang SM, Wilson CB. Treatment of progressive or recurrent glioblastoma multiforme in adults with herpes simplex virus thymidine kinase gene vector-producer cells followed by intravenous ganciclovir administration: A phase I/II multi-institutional trial. J Neurooncol 2003;65:269-78.

5. Rainov NG, Ren H. Clinical trials with retrovirus mediated gene therapy - what have we learned? J Neurooncol 2003;65:227-36.

6. Ren H, Boulikas T, Lundstrom K, Soling A, et al. Immunogene therapy of recurrent glioblastoma multiforme with a liposomally encapsulated replication-incompetent Semliki forest virus vector carrying the human interleukin12 gene-a phase I/II clinical protocol. J Neurooncol 2003;64:147-54.

7. Alavi JB, Eck SL. Gene therapy for high grade gliomas. Expert Opin Biol Ther 2001;1:239-52.

8. Grignet-Debrus C, Cool V, Baudson N, Velu T, et al. The role of cellular- and prodrug-associated factors in the bystander effect induced by the Varicella zoster and Herpes simplex viral thymidine kinases in suicide gene therapy. Cancer Gene Ther 2000;7:1456-68.

9. Nafe C, Cao YJ, Quinones A, Dobberstein KU, et al. Expression of mutant non-cleavable Fas ligand on retrovirus packaging cells causes apoptosis of immunocompetent cells and improves prodrug activation gene therapy in a malignant glioma model. Life Sci 2003;73:1847-60.

10. Alemany R, Gomez-Manzano C, Fueyo J. Oncolytic adenovirus for the treatment of cerebral tumors: Past, present and future. Neurologia 2001;16:122-7.

11. Rainov NG, Kramm CM, Banning U, Riemann D, et al. Immune response induced by retrovirus-mediated HSVtk/GCV pharmacogene therapy in patients with glioblastoma multiforme. Gene Ther 2000;7:1853-8.

12. Ikenaka K, Sasaki M, Tamura K, Tamura M, et al. Treatment of glioblastoma by direct inoculation of concentrated high titer-recombinant retrovirus carrying the herpes simplex virus thymidine kinase gene. Hum Cell 2001;14:49-58.

13. Marconi $\mathrm{P}$, Tamura M, Moriuchi S, Krisky DM, et al. Connexin 43-enhanced suicide gene therapy using herpesviral vectors. Mol Ther 2000;1:71-81.

14. Quillien V, Heresbach Le Berre N, Dufour T, Denais A, et al. Gene therapy of a model of glioblastoma in rats using adenovirus vector encoding the HSVtk gene. Bull Cancer 1997;84:1047-52.

15. Burrows FJ, Gore M, Smiley WR, Kanemitsu MY, et al. Purified herpes simplex virus thymidine kinase retroviral particles: III. Characterization of bystander killing mechanisms in transfected tumor cells. Cancer Gene Ther
2002;9:87-95.

16. Miura F, Moriuchi S, Maeda M, Sano A, et al. Sustained release of low-dose ganciclovir from a silicone formulation prolonged the survival of rats with gliosarcomas under herpes simplex virus thymidine kinase suicide gene therapy. Gene Ther 2002;9:1653-8.

17. Manome Y, Wen PY, Chen L, Tanaka T, et al. Gene therapy for malignant gliomas using replication incompetent retroviral and adenoviral vectors encoding the cytochrome P450 2B1 gene together with cyclophosphamide. Gene Ther 1996;3:513-20.

18. Ichikawa T, Petros WP, Ludeman SM, Fangmeier J, et al. Intraneoplastic polymer-based delivery of cyclophosphamide for intratumoral bioconversion by a replicating oncolytic viral vector. Cancer Res 2001;61:864-8.

19. Cho JY, Xing S, Liu X, Buckwalter TL, et al. Expression and activity of human $\mathrm{Na}+/ \mathrm{l}-$ symporter in human glioma cells by adenovirus-mediated gene delivery. Gene Ther 2000;7:740-9.

20. Aghi M, Kramm CM, Breakefield XO. Folylpolyglutamyl synthetase gene transfer and glioma antifolate sensitivity in culture and in vivo. J Natl Cancer Inst 1999;91:1233-41.

21. Tanaka T, Manome Y, Wen P, Kufe DW, et al. Viral vector-mediated transduction of a modified platelet factor 4 cDNA inhibits angiogenesis and tumor growth. Nat Med 1997;3:437-42.

22. De Bouard S, Guillamo JS, Christov C, Lefevre N, et al. Antiangiogenic therapy against experimental glioblastoma using genetically engineered cells producing interferonalpha, angiostatin, or endostatin. Hum Gene Ther 2003;14:883-95.

23. Harada H, Nakagawa K, Iwata S, Saito M, et al. Restoration of wild-type p16 down-regulates vascular endothelial growth factor expression and inhibits angiogenesis in human gliomas. Cancer Res 1999;59:3783-9.

24. Kim SK, Wang KC, Cho BK, Chung HT, et al. Interaction between $\mathrm{p} 53$ and $\mathrm{p} 16$ expressed by adenoviral vectors in human malignant glioma cell lines. J Neurosurg 2002;97:143-50.

25. Fueyo J, Gomez-Manzano C, Yung WK, Liu TJ, et al. Suppression of human glioma growth by adenovirus-mediated Rb gene transfer. Neurology 1998;50:1307-15.

26. Van Beusechem VW, Grill J, Mastenbroek DC, Wickham TJ, et al. Efficient and selective gene transfer into primary human brain tumors by using single-chain antibodytargeted adenoviral vectors with native tropism abolished. J Virol 2002;76:2753-62.

27. Weyerbrock A, Oldfield EH. Gene transfer technologies for malignant gliomas. Curr Opin Oncol 1999;11:168-73.

28. Shinoura N, Hamada H. Gene therapy using an adenovirus vector for apoptosis-related genes is a highly effective therapeutic modality for killing glioma cells. Curr Gene Ther 2003;3:147-53.

29. Shinoura N, Yamamoto N, Yoshida Y, Fujita T, et al. Adenovirus-mediated gene transduction of IkappaB or IkappaB plus Bax gene drastically enhances tumor necrosis factor (TNF)-induced apoptosis in human gliomas. Jpn J Cancer Res 2000;91:41-51.

30. Graf MR, Merchant RE. Interleukin-6 transduction of a rat T9 glioma clone results in attenuated tumorigenicity and induces glioma immunity in Fischer F344 rats. Neurooncol 1999;45:209-18.

31. Palu G, Cavaggioni A, Calvi P, Franchin E, et al. Gene 
therapy of glioblastoma multiforme via combined expression of suicide and cytokine genes: A pilot study in humans. Gene Ther 1999;6:330-7.

32. Parney IF, Farr-Jones MA, Kane K, Chang LJ, et al. Human autologous in vitro models of glioma immunogene therapy using B7-2, GM-CSF, and IL12. Can J Neurol Sci 2002;29:267-75.

33. Kikuchi T, Joki T, Saitoh $S$, Hata $Y$, et al. Anti-tumor activity of interleukin-2-producing tumor cells and recombinant interleukin 12 against mouse glioma cells located in the central nervous system. Int J Cancer 80:425-30.

34. Herrlinger U, Jacobs A, Quinones A, Woiciechowsky C, et al. Helper virus-free herpes simplex virus type 1 amplicon vectors for granulocyte-macrophage colonystimulating factor-enhanced vaccination therapy for experimental glioma. Hum Gene Ther 2000;11:1429-38.

35. Wakimoto $\mathrm{H}$, Yoshida $\mathrm{Y}$, Aoyagi M, Hirakawa $\mathrm{K}$, et al. Efficient retrovirus-mediated cytokine-gene transduction of primary-cultured human glioma cells for tumor vaccination therapy. Jpn J Cancer Res 1997;88:296-305.

36. Wang ZH, Zagzag D, Zeng B, Kolodny EH. In vivo and in vitro glioma cell killing induced by an adenovirus expressing both cytosine deaminase and thymidine kinase and its association with interferon-alpha. J Neuropathol Exp Neurol 1999;58:847-58.

37. Yamanaka R, Zullo SA, Ramsey J, Yajima N, et al. Marked enhancement of antitumor immune responses in mouse brain tumor models by genetically modified dendritic cells producing Semliki Forest virus-mediated interleukin-12. J Neurosurg 2002;97:611-8.

38. Ruan S, Okcu MF, Pong RC, Andreeff M, et al. Attenuation of WAF1/Cip1 expression by an antisense adenovirus expression vector sensitizes glioblastoma cells to apoptosis induced by chemotherapeutic agents 1,3-bis(2chloroethyl)-1-nitrosourea and cisplatin. Clin Cancer Res 1999;5:197-202.

39. Miller CR, Williams CR, Buchsbaum DJ, Gillespie GY. Intratumoral 5-fluorouracil produced by cytosine deaminase/5-fluorocytosine gene therapy is effective for experimental human glioblastomas. Cancer Res 2002;62:773-80.

40. Arafat WO, Buchsbaum DJ, Gomez-Navarro J, Tawil SA, et al. An adenovirus encoding proapoptotic Bax synergistically radiosensitizes malignant glioma. Int $\mathrm{J}$ Radiat Oncol Biol Phys 2003;55:1037-50.
41. Broaddus WC, Liu Y, Steele LL, Gillies GT, et al. Enhanced radiosensitivity of malignant glioma cells after adenoviral p53 transduction. J Neurosurg 1999;91:9971004.

42. Gridley DS, Baer JR, Cao JD, Miller GM, et al. TNF-alpha gene and proton radiotherapy in an orthotopic brain tumor model. Int J Oncol 21:251-9.

43. Gridley DS, Li J, Kajioka EH, Andres ML, et al. Combination of pGL1-TNF-alpha gene and radiation (proton and gamma-ray) therapy against brain tumor. Anticancer Res 2000;20:4195-203.

44. Lumniczky K, Desaknai S, Mangel L, Szende B, et al. Local tumor irradiation augments the antitumor effect of cytokine-producing autologous cancer cell vaccines in a murine glioma model. Cancer Gene Ther 2002;9:44-52.

45. Phuong LK, Allen C, Peng KW, Giannini C, et al. Use of a vaccine strain of measles virus genetically engineered to produce carcinoembryonic antigen as a novel therapeutic agent against glioblastoma multiforme. Cancer Res 2003;63:2462-9.

46. Gondi CS, Lakka SS, Yanamandra N, Siddique K, Dinh $\mathrm{DH}$, Olivero WC, et al. Expression of antisense UPAR and antisense uPA from a bicistronic adenoviral construct inhibits glioma cell invasion, tumor growth, and angiogenesis. Oncogene 2003;22:5967-75.

47. Cortes ML, de Felipe P, Martin V, Hughes MA, et al. Successful use of a plant gene in the treatment of cancer in vivo. Gene Ther 1998;5:1499-507.

48. Chiocca EA, Aghi M, Fulci G. Viral therapy for glioblastoma. Cancer J 2003;9:167-79.

49. Lang FF, Bruner JM, Fuller GN, Aldape K, et al. Phase I trial of adenovirus-mediated p53 gene therapy for recurrent glioma: Biological and clinical results. J Clin Oncol 2003;21:2508-18.

50. Trask TW, Trask RP, Aguilar-Cordova E, Shine HD, et al. Phase I study of adenoviral delivery of the HSV-tk gene and ganciclovir administration in patients with current malignant brain tumors. Mol Ther 2000;1:195-203.

51. Alemany R, Gomez-Manzano C, Balague C, Yung WK, et al. Gene therapy for gliomas: molecular targets, adenoviral vectors, and oncolytic adenoviruses. Exp Cell Res 1999;252:1-12.

52. Rainov NG, Ren H. Oncolytic viruses for treatment of malignant brain tumours. Acta Neurochir Suppl 2003;88:113-23. 